\title{
Lipid Spheres as Attractive Nanoscale Drug Delivery Platforms for Cancer Therapy
}

\section{Seang H. Yiv ${ }^{1,2}$ and Fatih M. Uckun ${ }^{1,2, *}$}

${ }^{1}$ Developmental Therapeutics Program, Children's Hospital Los Angeles, Children's Center for Cancer and Blood Diseases, Los Angeles, CA 90027, USA ${ }^{2}$ Department of Pediatrics, University of Southern California Keck School of Medicine, Los Angeles, CA 90027, USA

\section{Summary}

Several barriers limit the effective tumor delivery and penetration of the nanomedicine candidates as anti-cancer therapeutics, including: [1] heterogeneous tumor circulation caused by abnormal and irregular architecture of the tumor vasculature, [2] intratumoral vascular hyperpermeability contributing to increased interstitial pressure in the targeted tumor that substantially reduces the convective transport of nanoparticles and [3] impaired diffusion in the context of an abnormal and highly dense extracellular collagen matrix in the tumor microenvironment. Lipid spheres represent a promising drug formulation platform for effective delivery of anti-cancer pharmaceuticals to target cancer cells. Lipid spheres, especially microemulsion systems can be used to address various challenges in formulation and clinical use of anti-cancer pharmaceuticals. Due to a number of attributes inherent to lipid sphere nanoparticles, including their large lipid cores for the solubilization of anti-tumor agents, and the relative ease of lipid sphere manufacturing compared to the manufacturing of liposomes, lipid spheres have the potential to emerge as the preferred nanoscale delivery systems of anti-cancer therapeutics. Low Interfacial Tension Lipid Spheres (LIFT-LS) are classical thermodynamically stable microemulsions. LIFT of the nanoscale microemulsion systems and many of their LIFT-associated surface characteristics such as enhanced nanodroplet deformability, flexibility, and ease of emulsification at the contact point between the microemulsion nanodroplets and the target cancer cell membrane may contribute to an improved drug delivery capacity. The ability of the microemulsion nanodroplets to undergo shape changes and fragmentationcoalescence when subjected to environmental perturbations may further facilitate their transport and penetration through the extracellular tumor matrix despite high interstitial tissue pressure that exists inside solid tumors. Dilutable Low Interfacial Tension Lipid Spheres (DLIFT-LS) are a distinct subgroup of LIFT-LS and currently represent the most advanced form of microemulsions. It is our considered opinion that DLIFT-LS are the most suitable lipid spheres for intravenous anti-cancer drug delivery due to their multiple attributes as discussed in this review.

Keywords: Nanomedicine; Microemulsion; Cancer; Oncology; Formulation; Drug delivery

\section{Introduction}

Nanotechnology-enabled delivery of anti-cancer therapeutics is an area of intense translational research [1-14]. Rationally designed biotargeted anti-cancer nanomedicines have the potential to substantially improve the therapeutic index of their "payload" by (1) increasing their potency via (a) selective delivery to target cancer cells as well as (b) improved cellular pharmacokinetic/pharmacodynamic (PK/PD) features that avoid the multi-drug resistance associated drug efflux pumps and (2) reducing their systemic toxicity and undesired offtarget effects. Several non-targeted nanomedicine candidates are being evaluated in clinical trials or have been given FDA approval, including biocompatible micellar, liposomal, and polymeric formulations of standard chemotherapy drugs (e.g. camptothecin, doxorubicin, paclitaxel, cisplatin). Several biotargeting moieties are being explored in pre-clinical studies including small molecules, antibodies/antibody fragments, affibodies, cell penetrating peptides, cytokines, avimers and aptamers.

A nanoscale formulation platform must exhibit many of the following attributes for it to be a suitable drug delivery system for cancer therapy:

1. Capacity to encapsulate, entrap, or solubilize potent anti-cancer drug substances

2. Ability to escape the reticuloendothelial system (RES), remain stable in blood circulation as well as normal tissue microenvironment and retain the active drug substance as payload until the target tumor site is reached
3. Ability to penetrate the physical barriers of the tumor extracellular matrix and reach cancer stem cells

4. Ability to be rapidly internalized by cancer cells and release the payload upon internalization.

Among nanoscale formulation platforms, lipid spheres, including microemulsions and micellar preparations, are composed of a lipid core surrounded and stabilized by a surface monolayer of surfactant. Figure 1 illustrates the similarities and differences of various lipid sphere systems. The thermodynamic and dynamic states of these systems affect their suitability as intravenous drug delivery vehicles. Microemulsions $[15,16]$ are isotropic, thermodynamically stable systems of oil, water and surfactant with a 20-100 nm droplet size. They can be prepared as oilin-water $(\mathrm{o} / \mathrm{w})$, water-in-oil (w/o) or bicontinuous systems depending on the equilibrium spontaneous curvature of the surfactant layer at the oil-water interface [36]. Thermodynamically stable microemulsions

*Corresponding author: Fatih M. Uckun, Children's Center for Cancer and Blood Diseases, Childrens Hospital Los Angeles, MS\#57, Los Angeles, California 90027 0367, USA, Tel: 323-361-4328; Fax: 323-361-876; E-mail: fmuckun@chla.uhsc. edu

Received December 22, 2011; Accepted January 04, 2012; Published January 06, 2012

Citation: Yiv SH, Uckun FM (2012) Lipid Spheres as Attractive Nanoscale Drug Delivery Platforms for Cancer Therapy. J Nanomedic Nanotechnol 3:128. doi:10.4172/2157-7439.1000128

Copyright: (c) 2012 Yiv SH, et al. This is an open-access article distributed under the terms of the Creative Commons Attribution License, which permits unrestricted use, distribution, and reproduction in any medium, provided the original author and source are credited. 

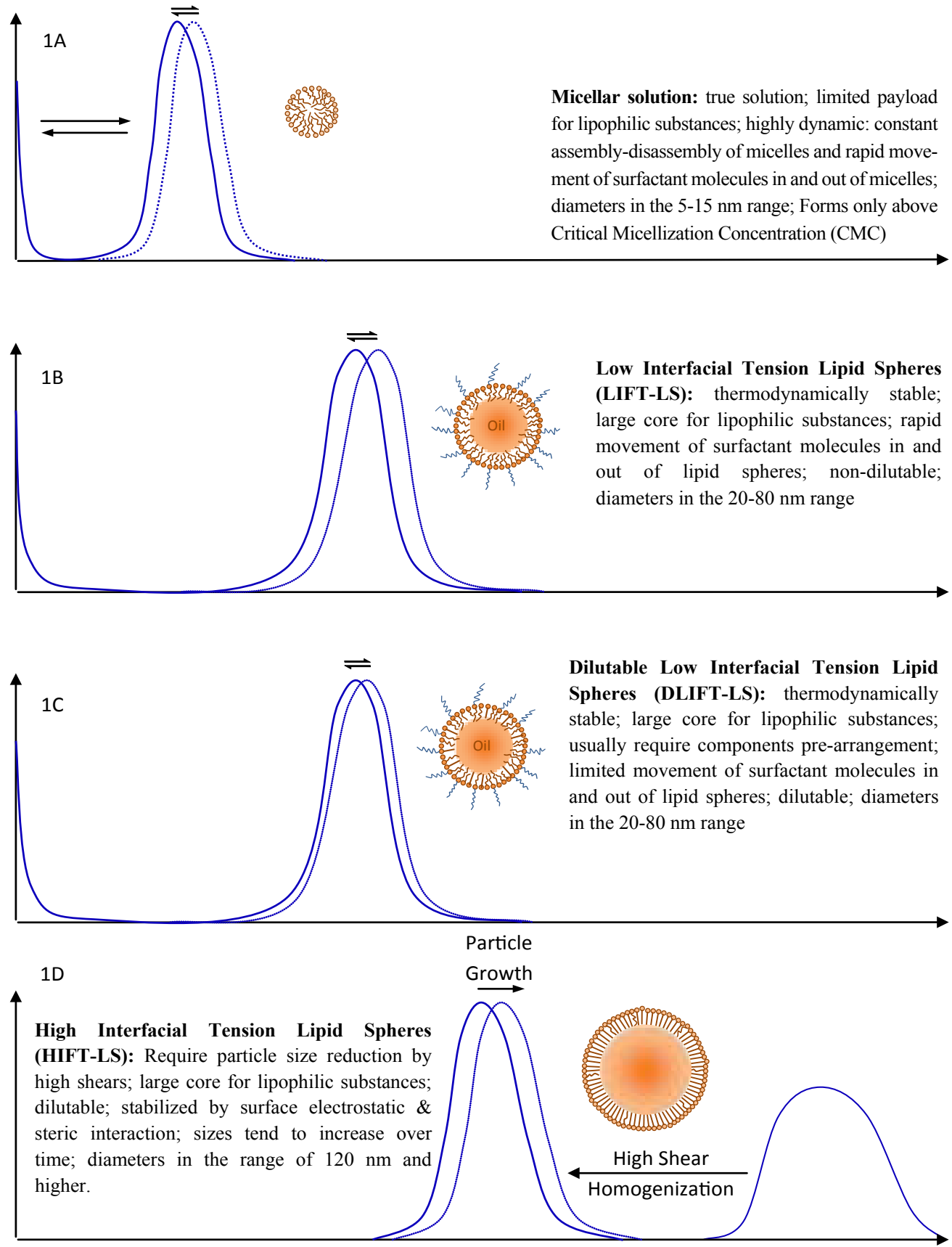

Figure 1: Similarities and differences between micellar solutions, microemulsions, and emulsions.

are characterized by the existence of ultra-low interfacial tension (LIFT) between oil and water phases. In this review, we will discuss the characteristics and clinical potential of lipid spheres, including oilin-water microemulsion systems, as intravenous drug delivery vehicles.

\section{Lipid Spheres}

Lipid spheres represent a promising drug formulation platform for effective delivery of anti-cancer pharmaceuticals to target cancer cells [52-55]. Lipid spheres can be used to address various challenges in formulation and clinical use of anti-cancer pharmaceuticals. Lipid spheres have been used as solubilization enhancement carriers for anticancer therapeutics (e.g. paclitaxel) [46-55]. Furthermore, lipid spheres have been shown to contribute to significantly improved tolerability of locally irritant pharmaceuticals. Intravenous administration of active drug substances using lipid sphere formulation platforms has been associated with reduced risk of inflammation and pain at injection sites [49-51].

\section{Micellar solutions (MS)}

In classical MS, lipids including lipid-soluble bio-active agents are solubilized within the core and/or the surfactant surface layer. The main defining parameters of MS, namely the critical micelle concentration (CMC) (i.e., threshold concentration of the surfactant above which the micelles begin to form) and the micellization number (i.e., the number of surfactant molecules per micelle) are largely determined by the 
structure of the surfactant (hydrophilic and hydrophobic moieties) and to a lesser extent other factors such as ionic strength of the aqueous environment. Because of their small size, MS have a relatively limited capacity for the solubilization or incorporation of bio-active molecules with amphiphilic or lipophilic properties (Figure 1A). MS are highly dynamic systems $[17,18]$ : The individual surfactant molecules freely and continuously exit and enter the micelles according to a very fast kinetics with a nanosecond time scale. Likewise, the micelles themselves disintegrate and re-assemble quickly according to a milliseconds to seconds time scale. This very dynamic feature of MS causes them to be highly surface reactive, promotes the formation of air bubbles and may contribute to embolic complications after intravenous administration.

\section{Amphiphilic block copolymer micelles}

Several non-traditional MS comprised of amphiphilic block copolymers $(\mathrm{ABC})$ have been reported. Some of them do not have the disadvantage of fast kinetics of classical MS containing small surfactant molecules [2,4,19-25]. ABCs are more versatile and suitable for drug delivery applications than (a) classical small surfactant molecules owing to their lower CMC values and greater stability as well as (b) classical micelles owing to their less lipophilic core which can be designed to accommodate charged drug substance molecules via ionic interactions. One ABC drug delivery formulation made of polyethylene glycol and polypropylene glycol block copolymers (PEO-PPO-PEO) has been in clinical trial [26] whereas several new generation $A B C$ s have either received regulatory approval or entered proof-of-principle clinical testing [27-31].

\section{Low interfacial tension lipid spheres (LIFT-LS)}

LIFT-LS are classical thermodynamically stable microemulsions, which form readily upon mixing of their components, but they are generally not amenable to dilution (Figure 1B) [15-18]. In addition, most of the reported LIFT-LS have not been prepared using pharmaceutically acceptable components. Because of their large lipid core, LIFT-LS do not disintegrate as readily as micelles. Rather, they display some fluctuation of their size secondary to fragmentationcoalescence, which occurs through fusion-fission of the particles (Figure 2). Fusion-fission is a unique feature of these microemulsion systems due to their LIFT. The presence of low IFT is best documented by using a phase diagram $[33,34]$ (Figure 3). LIFT, including ultra-low IFT with IFT values 3 orders of magnitude lower than the oil-water interfacial tension, can be determined directly by using a spinning drop technique [35]. In addition, through a systematic, simple but careful change in the surfactant structure, such as the branching of the lipophilic tail and change of the head group size, cosurfactant-free microemulsions with very high solubilization of internal phase (as high as 1 to 30 of surfactant to internal phase weight ratio) can be obtained [34]. This latter feature is especially beneficial for the solubilization and delivery of poorly soluble anti-cancer therapeutics. In addition, LIFT of the nanoscale microemulsion systems and many of their LIFT-associated surface characteristics such as enhanced nanodroplet deformability, flexibility, and ease of emulsification at the contact point between the microemulsion nanodroplets and the target cancer cell membrane may contribute to an improved drug delivery capacity [36-38]. The near zero or even negative free energy of the microemulsion compositions favors spontaneous emulsification, near zero spontaneous interfacial curvature, ultra-low IFT, small particle size, and high solubilization [36-38]. The ability of the microemulsion nanodroplets to undergo shape changes and fragmentation-coalescence when subjected to environmental perturbations, may further facilitate their transport and penetration through the extracellular tumor matrix despite high interstitial tissue pressure that exists inside solid tumors [1,32]. In addition, these features may also enhance their cellular internalization [6]. It is worth noting that these favorable characteristics for drug delivery are inherent only to LIFT microemulsion systems and do not apply to emulsion and liposome nanoparticles.

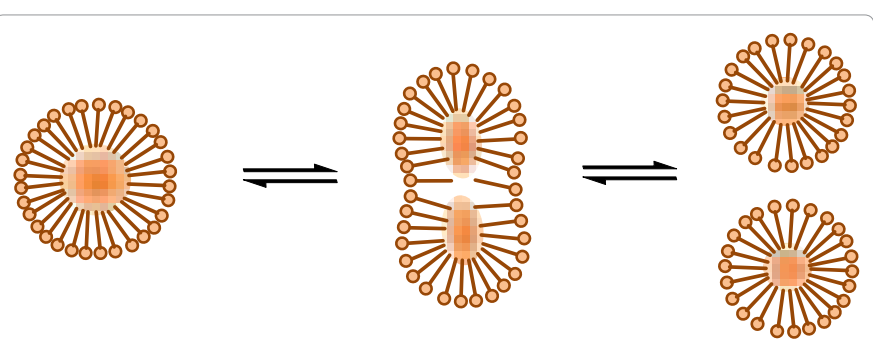

Figure 2: Representation of microemulsion nanodroplet Fusion-Fission.

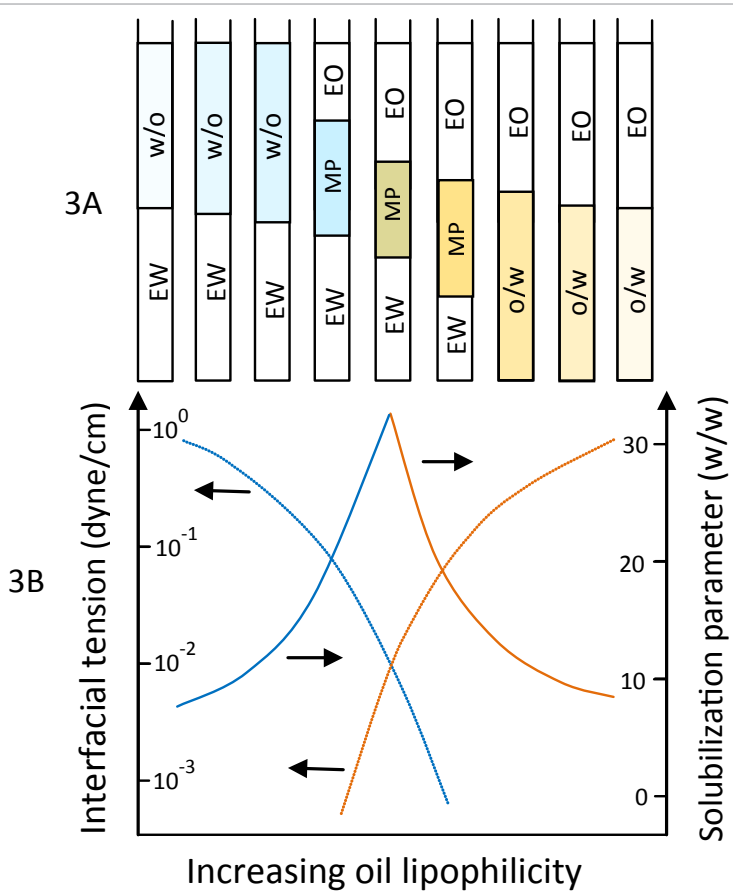

Figure 3: Changes in phase behavior $(3 \mathrm{~A})$, solubilization, and interfacial tension $(3 \mathrm{~B})$ in a microemulsion system with increasing oil lipophilicity. w/o: Water-inoil microemulsion; o/w: Oil-in-water microemulsion; MP: Middle phase (can be $\mathrm{w} / \mathrm{o}, \mathrm{o} / \mathrm{w}$, or bicontinuous); Solubilization parameter is the ratio of the internal phase to the surfactant concentration. Increasing oil lipophilicity can be made by increasing the alkane carbon number (ACN) of hydrocarbon (for example from hexane to hexadecane). From left to right, a transition is seen from w/o microemulsion in equilibrium with excess water (EW), to MP in equilibrium with both $\mathrm{EW}$ and excess oil (EO), to o/w microemulsion in equilibrium with EO. In the w/o region, the solubilization of water in oil increases from left to right. The solubilization is maximal in the three phase region. In the o/w region, the solubilization of the oil decreases further from the three phase region and toward higher ACN. Other convenient variables that can be changed include temperature (for nonionic surfactant in particular), salt concentration, etc. Maximum solubilizations occur near and at the transition point: $w / o \rightarrow$ MP region $\rightarrow$ o/w (3A), where the lowest interfacial tensions also occur (3B).

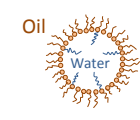

Addition of buffer with gentle mixing Inversion/Conversion

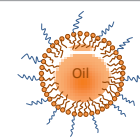

Water 


\section{Dilutable low interfacial tension lipid spheres (DLIFT-LS)}

DLIFT-LS are a distinct subgroup of LIFT-LS [39] and currently represent the most advanced form of microemulsions (Figure 1C). They can be obtained readily by diluting a concentrated microemulsion preparation or by using a standard conversion/inversion technique (e.g conversion from a w/o to an o/w system) (Figure 4). DLIFT-LS are significantly less dynamic than LIFT-LS and the movements of the individual surfactant molecules in and out DLIFT-LS are very limited and much slower. It is our considered opinion that DLIFT-LS are the most suitable lipid spheres for intravenous anti-cancer drug delivery due to their multiple attributes listed in Table 1.

\section{Traditional Emulsions or High Interfacial Tension Lipid Spheres (HIFT-LS)}

HIFT-LS are emulsions that are prepared using high shear homogenization (Figure 1D). Most commercial lipid emulsions utilize soy or egg lecithin as the primary surfactant. Some experimental emulsions have been obtained by means of solvent injection without using high shear pressure. However, although these latter lipid spheres can be produced without high pressure equipment, there is no certainty that they will reproducibly and regularly form under LIFT conditions. The interfacial tension of HIFT-LS is at least one order of magnitude higher than that of LIFT-LS. The best known HIFT-LS are intravenous lipid emulsions such as Intra-lipid. They are not thermodynamically stable and their particle size tends to increase over time [40-43]. However, when properly formulated and manufactured, the presence of electrostatic interactions at the interface provides sufficient stabilization for the particles to slow the particle growth during storage and their stability is therefore pharmaceutically acceptable [40-43].

\section{Excipients Suitable for Intravenous Lipid Spheres}

The lipophilic phase, which makes up the internal core, and the surface active agents (surfactants), which surround and stabilize the internal lipophilic core are the two main components of lipid spheres. Most research and development on preparation of microemulsions during the last 4 decades was performed using industrial excipients that are pharmaceutically not acceptable. Therefore, the identification of alternative excipients, especially suitable lipid and surfactant substitutes, has emerged as an area of intense investigation in formulation research for delivery of bioactive molecules in nanoscale lipid spheres. Such excipients must be (a) inert, (b) non-toxic or "generally regarded as safe" (GRAS), (c) pharmaceutical grade, (d) available in commercial quantities, and (e) suitable for intravenous administration.

\section{Surfactants and cosurfactants}

Many of the currently available surfactants are suitable for intravenous use [44]. One of the most acceptable components for pharmaceutical lipid spheres is lecithin, which is a key component of the cell wall. Phospholipids can be formulated with other components to form either liposomes or lipid spheres, depending on whether or not oil is present in the formulation. They are among the most versatile and enabling excipients in liposome and lipid sphere chemistry and can be used in the preparation of rationally designed multifunctional nanoparticles. Because microemulsion formulations can only be formed with surfactants or surfactant combinations having an appropriate hydrophilic lipophilic balance (HLB) for a given lipophilic phase, the use of a second surfactant besides lecithin often becomes necessary. The available options include surfactants with high HLBs such as PEGylated phospholipids. PEGylated phospholipids are double tail surfactants with a long polyethylene head group and they maintain their stability upon dilution due to their low CMC. Their micelles are capable of solubilizing large quantities of lipophilic bio-active molecules. PEGylated phospholipids have been used as an excipient in the FDA-approved liposomal formulation of the standard anticancer chemotherapy drug doxorubicin, Doxil [45]. Cosurfactants may also be needed for some formulations, but many of the available cosurfactants (e.g. short chain alcohols) are not suitable for clinical use because of their toxicity. Most suitable microemulsions for intravenous administration are therefore those that can be formulated without the need of a cosurfactant.

\section{Lipid phase}

There are a large number of lipids, especially triglycerides that can be used as the core component of lipid spheres [44]. However, for the microemulsions to be useful as nanotechnology-enabled intravenous drug delivery systems, their lipid core must serve as an excellent solubilizer for the pharmaceutical "payload". The best known lipids used in preparation of lipid spheres are long chain triglycerides, medium chain triglycerides (MCT), and vitamin E [39-55]. By using a phase diagram, the total lipid core volume of microemulsions can be maximized in an effort to optimize the solubilization of bio-active molecules to be delivered to target cancer cells [33-34,39].

\section{Expert Opinion}

Several barriers limit the effective tumor delivery and penetration of the nanomedicine candidates as anti-cancer therapeutics, including: (1) heterogeneous tumor circulation caused by abnormal and irregular

\begin{tabular}{|c|c|c|}
\hline 1 & Small particle size & $\begin{array}{l}\text { Because of their small particle size }(20-100 \mathrm{~nm}) \text {, DLIFT-LS are suitable as intravenous delivery carriers, } \\
\text { expected to exhibit an in vivo EPR behavior similar to that of liposomes }\end{array}$ \\
\hline 2 & Solubilization of lipophilic drugs & $\begin{array}{l}\text { Due to the presence of large lipophilic/amphiphilic domains within their structures, DLIFT-LS have an } \\
\text { excellent capacity for the solubilization of lipophilic/amphiphilic drug molecules. }\end{array}$ \\
\hline 3 & Stealth behavior & $\begin{array}{l}\text { Because DLIFT-LS usually contain significant quantities of PEGylated surfactants, they are expected to } \\
\text { display the shared "stealth" behavior of PEGylated nanoparticles. }\end{array}$ \\
\hline 4 & No need for high shear equipment & $\begin{array}{l}\text { DLIFT-LS form spontaneously or with gentle mixing. Therefore, only conventional mixing equipment is } \\
\text { needed for their manufacturing }\end{array}$ \\
\hline 5 & Can be filtered or heat sterilized & $\begin{array}{l}\text { DLIFT-LS can easily be filtered through } 0.22 \mu \mathrm{m} \text { filter. During heat sterilization, phase separation may } \\
\text { occur, but DLIFT-LS should return to their original state when cooling to ambient temperature }\end{array}$ \\
\hline 6 & Dilutable & Dilutability uniquely distinguishes DLIFT-LS from other microemulsion systems. \\
\hline 7 & Long term storage at $4^{\circ} \mathrm{C}$ & A broad temperature stability range $\left(4-50^{\circ} \mathrm{C}\right)$ allows the DLIFT-LS to be stored long-term at $4^{\circ} \mathrm{C}$ \\
\hline 8 & Possible enhanced fusion with cell membrane & $\begin{array}{l}\text { Because of their LIFT and small particle size, the internalization of DLIFT-LS nanodroplets by target } \\
\text { cancer cells may be further facilitated by fusion with cancer cell membrane }\end{array}$ \\
\hline 9 & Antibody/Ligand Insertion & $\begin{array}{l}\text { Insertion of a targeting antibody or ligand into the surface of the DLIFT-LS can be accomplished as a } \\
\text { last step during their preparation }\end{array}$ \\
\hline
\end{tabular}

Table 1: Attributes of DLIFT-LS as a tumor targeting platform 
architecture of the tumor vasculature, (2) intratumoral vascular hyperpermeability contributing to increased interstitial pressure in the targeted tumor that substantially reduces the convective transport of nanoparticles and (3) impaired diffusion in the context of an abnormal and highly dense extracellular collagen matrix in the tumor microenvironment. Therefore, development of innovative strategies aimed at correcting the abnormal tumor vasculature and matrix as well as rational design of novel nanomedicines, including multistage delivery systems capable of overcoming the physiological tumor tissue barriers have emerged as focal points in contemporary translational research. In parallel, efforts are underway to optimize and control the multistep manufacturing process of nanomedicine candidates for efficient and reproducible self-assembly of functional nanoparticles with more precise control of pharmacodynamically relevant physical/ chemical parameters of the nanoparticles.

Although lipid spheres have been used as intravenous drug carriers, drug solubilization enhancement vehicles, and suppressors of injection site irritation, their full potential as nanoscale anti-cancer drug delivery systems remains to be explored. When the small size of micelles becomes a limiting factor for the delivery of a therapeutic payload, a suitable DLIFT-LS delivery system can be rationally designed by using a phase diagram or phase change construction. Due to a number of attributes inherent to lipid sphere nanoparticles, including their large lipid cores for the solubilization of anti-tumor agents, and the relative ease of lipid sphere manufacturing compared to the manufacturing of liposomes, lipid spheres have the potential to emerge as the preferred nanoscale delivery systems of anti-cancer therapeutics.

\section{Ackowledgment}

This work was supported in part by DHHS Grant No. U01-CA-151837

\section{References}

1. Jain RK, Stylianopoulos T (2010) Delivering nanomedicine to solid tumors. Nat Rev Clin Oncol 7: 653-664.

2. Torchilin VP (2007) Micellar nanocarriers: Pharmaceutical perspectives. Pharm Res 24: 1-16.

3. Nie S (2010) Understanding and overcoming major barriers in cancer nanomedicine. Nanomedicine 5: 523-528.

4. Lindman B, Alexandridis $P$ (2000) Amphiphilic block copolymers: self-assembly and applications. Elsevier Science.

5. Davis ME, Chen ZG, Shin DM (2008) Nanoparticle therapeutics: an emerging treatment modality for cancer. Nat Rev Drug Discov 7: 771-782.

6. Petros RA, DeSimone JM (2010) Strategies in the design of nanoparticles for therapeutic applications. Nat Rev Drug Disco 9: 615-627.

7. Pouton CW, Wagstaff KM, Roth DM, Moseley GW, Jans DA (2007) Targeted delivery to the nucleus. Adv Drug Deliv Rev 59: 698-717.

8. Ferrari M (2005) Cancer nanotechnology: opportunities and challenges. Nat Rev Cancer 5: 161-171.

9. Brewer E, Coleman J, Lowman A (2011) Emerging technologies of polymeric nanoparticles in cancer drug delivery. J Nanomaterials.

10. Guo S, Huang L (2011) Nanoparticles escaping RES and endosome: challenges for siRNA delivery for cancer therapy. J Nanomaterials.

11. Tiwari PM, Vig K, Dennis VA, Singh SR (2011) Functionalized gold nanoparticles and their biomedical applications. Nanomaterials 1: 31-63.

12. Zhang L, Gu FX, Chan JM, Wang AZ, Langer RS, et al. (2008) Nanoparticles in medicine: therapeutic applications and developments. Clin Pharmacol Ther 83: 761-769

13. Bharali DJ, Khalil M, Gurbuz M, Simone TM, Mousa SA (2009) Nanoparticles and cancer therapy: a concise review with emphasis on dendrimers. Int $J$ Nanomedicine 4: 1-7.
14. Talegaonkar S, Azeem A, Ahmad FJ, Khar RK, Pathan SA, et al. (2008) Microemulsions: a novel approach to enhanced drug delivery. Recent Pat Drug Deliv Formul 2: 238-257

15. Hoar TP, Schulman JH (1943) Transparent water-in-oil dispersions: the oleopathic hydro-micelle. Nature 152: 102-103.

16. Schulman JH, Stoeckenius H, Prince LM (1959) Mechanism of formation and structure of microemulsions by electron microscopy. J Phys Chem 63: 16771680

17. Aniansson EAG, Wall SN (1974) Kinetics of step-wise micelle association. J Phys Chem 78: 1024-1030.

18. Zana R (2005) Dynamics of Surfactant Self-Assemblies: Micelles, Microemulsions, Vesicles and Lyotropic Phases. CRC Press.

19. Savić R, Luo L, Eisenberg A, Maysinger D (2003) Micellar nanocontainers distribute to defined cytoplasmic organelles. Science 300: 615-618.

20. Pounder RJ, Willcock H, leong NS, O'Reilly RK, Dove AP (2011) Stereocomplexation in novel degradable amphiphilic block copolymer micelles of poly(ethylene oxide) and poly(benzyl a-malate). RSC Soft Matter 7: 1098710993.

21. Kwon GS, Forrest ML (2006) Amphiphilic block copolymer micelles for nanoscale drug delivery. Drug Development Research 67: 15-22.

22. Mikhail AS, Allen C (2009) Block copolymer micelles for delivery of cancer therapy: Transport at the whole body, tissue and cellular levels. J Control Release 138: 214-223.

23. Gao ZG, Fain HD, Rapoport N (2005) Controlled and targeted tumor chemotherapy by micellar-encapsulated drug and ultrasound. J Contro Release 102: 203-222.

24. Licciardi M, Giammona G, Du J, Armes SP, Tang Y, et al. (2006) New folatefunctionalized biocompatible block copolymer micelles as potential anti-cancer drug delivery systems. Polymer 47: 2946-2955.

25. Nishyama N, Kataoka K (2003) Polymeric micelle drug carrier systems: PEGPAsp (Dox) and second generation of micellar drugs. Polymer Drugs in the Clinical Stage 519: 155-177.

26. Kabanov AV, Batrakova EV, Alakhov VY (2002) Pluronic block copolymers for overcoming drug resistance in cancer. Adv Drug Deliv Rev 54: 759-779.

27. Kim DW, Kim SY, Kim HK, Kim SW, Shin SW, et al. (2007) Multicenter phase II trial of Genexol-PM, a novel Cremophor-free, polymeric micelle formulation of paclitaxel, with cisplatin in patients with advanced non-small-cell lung cancer. Ann Oncol 18: 2009-2014.

28. Kim J, Shin S (2010) Cost-effectiveness of Genexol-PM for treating metastatic breast cancer. J Breast Cancer 13: 104-110.

29. Lee SW, Yun MH, Jeong SW, In CH, Kim JY, et al. (2011) Development of docetaxel-loaded intravenous formulation, Nanoxel-PM ${ }^{\mathrm{TM}}$ using polymerbased delivery system. J Control Release 155: 262-71.

30. Matsumura Y, Hamaguchi T, Ura T, Muro K, Yamada Y, et al. (2004) Phase clinical trial and pharmacokinetic evaluation of NK911, a micelle-encapsulated doxorubicin. Br J Cancer 91: 1775-1781.

31. Hamaguchi T, Kato K, Yasui H, Morizane C, Ikeda M, et al. (2007) A phase I and pharmacokinetic study of NK105, a paclitaxel-incorporating micellar nanoparticle formulation. Br J Cancer 97: 170-176.

32. Narang AS, Varia S (2011) Role of tumor vascular architecture in drug delivery. Adv Drug Deliv Rev 63: 640-658.

33. Heyes ME, Bourrel MU, El-Emary MM, Schechter RS, Wade WH (1979) Interfacial tension and behavior of nonionic surfactants. SPE Journal 19: 349356 .

34. Abe M, Schechter D, Schechter RS, Wade WH, Weerasooriya U, et al (1986) Microemulsion formation with branched tail polyoxyethylene sulfonate surfactants. J Colloid Interface Sci 114: 342-356.

35. Cayias JL, Schechter RS, wade WH (1975) The Measurement of Low Interfacia Tension via the Spinning Drop Technique. Adsorption at Interfaces 8: 234-237.

36. DeGennes PG, Taupin C (1982) Microemulsions and the flexibility of oil/water interfaces. J Phys Chem 86: 2294-2304.

37. Talmon Y, Prager S (1978) Statistical thermodynamics of phase equilibria in microemulsions. J Chern Phys 69: 2984-2991. 
Citation: Yiv S, Uckun FM (2012) Lipid Spheres as Attractive Nanoscale Drug Delivery Platforms for Cancer Therapy. J Nanomedic Nanotechnol 3:128. doi:10.4172/2157-7439.1000128

38. Nagarajan R, Ruckenstein E (2000) Molecular theory of microemulsions. Langmuir 16: 6400-6415.

39. Yiv SH, Tustian AK (2001) Drug delivery compositions suitable for intravenous injection, United States Patent 6,245,349 B1.

40. Zaloga GP, Dunham AJ, Gonyon TM, Best LM (2007) Safety and stability of lipid emulsions. Nutr Clin Pract 22: 367-369.

41. Rey JB, Faure C, Brion F (2005) Stability of all-in-one standard formulae for paediatric parenteral nutrition. PDA J Pharm Sci Tech 59: 206-220.

42. Mehta RC, Head LF, Hazrati AM, Parr M, Rapp RP, et al. (1992) Fat emulsion particle-size distribution in total nutrient admixtures. Am J Hosp Pharm 49: 2749-2755.

43. Deitel M, Friedman KL, Cunnane S, Lea PJ, Chaiet A, et al. (1992) Emulsion stability in a total nutrient admixture for total parenteral nutrition. J Am Coll Nutr 11: $5-10$.

44. Strickley RG (2004) Solubilizing excipients in oral and injectable formulations. Pharm Res 21: 201-230.

45. skubitz km (2002) Phase II trial of pegylated-liposomal doxorubicin (doxil) in mesothelioma. Cancer Invest 20: 693-699.

46. Wang Y, Wu KC, Zhao BX, Zhao X, Wang X, et al. (2011) A novel paclitaxel microemulsion containing a reduced amount of cremophor el: pharmacokinetics, biodistribution, and in vivo antitumor efficacy and safety. J Biomed Biotechnol.

47. Ryoo HK, Park CW, Chi SC, Park ES (2005) Development of propofol loaded microemulsion systems for parenteral delivery. Arch Pharm Res 28: 1400-140.
48. Park KM, Kim CK (1999) Preparation and evaluation of flurbiprofen-loaded microemulsion for parenteral delivery. Int J Pharm 181: 173-179.

49. Date AA, Nagarsenker MS (2008) Design and evaluation of microemulsions for improved parenteral delivery of propofol. AAPS PharmSciTech 9: 138-145.

50. Dubey PK, Kumar A (2005) Pain on injection of lipid-free propofol and propofo emulsion containing medium-chain triglyceride: a comparative study. Anesth Analg 101: 1060-1062.

51. Lee JM, Park KM, Lim SJ, Lee MK, Kim CK (2002) Microemulsion formulation of clonixic acid: solubility enhancement and pain reduction. J Pharm Pharmacol 54: 43-49.

52. Wang J, Maitani Y, Takayama K (2002) Antitumor effects and pharmacokinetics of aclacinomycin A carried by injectable emulsions composed of vitamin $E$, cholesterol, and PEG-lipid. J Pharm Sci 91: 1128-1134.

53. Junping W, Takayama K, Nagai T, Maitani $Y$ (2003) Pharmacokinetics and antitumor effects of vincristine carried by microemulsions composed of PEGlipid, oleic acid, vitamin E and cholesterol. Int J Pharm 251: 13-21.

54. Wang JP, Wang W, Zhao LN (2009) Paclitaxel carried by long circulating microemulsions used for metronomic chemotherapy of cancer. Yao Xue Xue Bao 44: 911-914.

55. Shiokawa T, Hattori Y, Kawano K, Ohguchi Y, Kawakami H, et al. (2005) Effect of polyethylene glycol linker chain length of folate-linked microemulsions loading aclacinomycin a on targeting ability and antitumor effect in vitro and in vivo. Clin Cancer Res 11: 2018-2025. 